\section{THE BRADSHAW LECTURE ON}

\section{THE INFLUENCE OF THE SYMPATHETIC ON DISEASE.}

\author{
Delivered before the Royal College of Physicians of London, \\ August I8th, I882. \\ BY EDWARD LONG FOX, M.D., F.R.C.P., \\ Consulting Physician to the Bristol Royal Infirmary.
}

Whes you, sir, did me the honour of inviting me to deliver this lecture here to-day, I was tempted to the choice of a subject that is not only a vast one, but also, perhaps, somewhat indefinite.

Anyone who has worked for many years in the science and art of medicine must feel some diffidence in expressing views that may be open to controversy, before any hody of men so highly educated as are the members of the medical profession at the present time. But this diffidence is necessarily rendered greater, when his immediate audience are persons closely connected with this great College; and, although this feeling must obtain with reference to all lines of thought connected with medical science, it is very specially the case with regard to the subject of this lecture, the Influence of the Sympathetic System on Disease, inasmuch as, without depreciating the earnest investigations carried out in Germany, in France, in Italy, in Russia, on the physiology and pathology of this portion of the nervous system, yet much of the best observation on the subject, much of the best reasoning on what may be called sympathetic phenomena, based on clinical and pathological data, has been placed before the profession at large by Fellows and Members of this College of Physicians. And if to-day the mention by name of any observer be perchance omitted, it will not be from want of due recognition on my part, but either because independent observations have been made in this, as in other scientific questions, almost synchronously in different countries, but chiefly that the results of many investigations have become, like those of Claude Bernard, household words amongst us. One word more I should like to say. Some years ago, the sympathetic system was the subject of the Astley Cooper prize at Guy's Hospital. Although the prize essay has never been published, by the kind permission of the author, Mr. George Arthur Woods of Southport, and the extreme courtesy of the authorities at Guy's, I have been favoured with a perusal of it ; and it is only right thus publicly to express my obligations.

The influence of the sympathetic system on disease must necessarily depend on its functional position in the economy. Is it in any sense an independent system? Has it functions of itself? Do its ganglia own a power possessed by no other portion of the frame, disconnected from any other system? or is the sympathetic only a nervous organ of conduction, carrying impressions from the internal viscera to the cerebro-spinal system, conducting to these internal viscera, and to the vessels, orders from the higher nervous centres? Are the sympathetic ganglia only the central formation from which peripheral nerves are formed, and the residue left after their formation? Is the sympathetic, in Hermann's words, nothing else than a branched roadway from the cerebro-spinal system, into which overflow paths of innervation from all points of the great nerve-centres, to spread to all sides of the periphery?

Did the sympathetic possess no intrinsic independence, it would be idle to speak of its influence on disease, except in so far as its connecting lines of fibres were compressed, or their conducting power otherwise interfered with. But bound up together as all nerve-tissue in the body is, the fœetus has been expelled from the uterus at, or almost at, full time, showing therefore, a normal capacity of absorbing nutrition, and a healthy circulation, without any trace of a cerebrospinal nervous centre, owning only the sympathetic system as the nerve-element in its composition.

The opinion of Claude Bernard that the sympathetic ganglia are real nervous centres, that the sympathetic is the motor nerve of the circulation, that the vaso-motor system is the regulator of heat, of nutrition, and of force ; the dictum of Goltz that the tone of the arteries is maintained by local centres, situated in their own immediate vicinity, and that it is wholly independent of the cerebro-spinal axis ; the fact mentioned by Woods, that reflex irritation of vaso-motor nerves (unlike reflex irritation of cerebro-spinal nerves) is entirely limited to the particular tissue or organs supplied, so that these nerve-fibres must have their centres in the sympathetic ganglia; the statement of Parkes, that nutrition is perfectly carried on with complete destruction of the cerebro-spinal centres, are all founded on observations or experiments that cannot be controverted.

Seeing, too, the impossibility of tracing a fibre of Remak in the tissue of the brain or spinal cord, the existence of vaso-motor centres in these organs, proved as it is by vaso-motor disturbances on lesions of certain districts of the cerebro-spinal centres, affords by no means positive proof of sympathetic ganglia being directly fed and influenced by them. The sympathetic connection between the medulla oblongata and the two chief centres in the cervical cord, the vaso-motor centre for the head and face, and the oculo-pupillary, is proved by experiment and by pathology. Such centres may lie in the cord and yet not be of it; and the whole system consists not only of the well known chains of ganglia, of the nerves and plexuses of the internal organs, of a vasomotor apparatus for the whole body, but of important ganglia that are situated, probably for purposes of correlation, within the tissues of the great centres of the cerebro-spinal system.

The possible independence of the sympathetic is shown by an experiment of Vulpian. He found that, some days after the transverse section of the sciatic nerve, or of the brachial plexus, when the corresponding pulps of the paws of the animal had become quite pale and anæmic, one might, by slight rubbing of these pulps, cause a reflex congestion. This vaso-dilator reflex effect seemed to the author to prove the existence of peripheral nervous centres, ganglia, "land"nervous-cells in relation with the vaso-motor nerve-fibres.

And this leads to one more preliminary remark-viz., that certain phenomena seem to prove indubitably the existence of vaso-dilators. Not only must Claude Bernard's discovery of this property in the chorda tympani, and Eckhart's in the nervi erigentes, be accepted as proof of this view, but it is more than probable that all the centrifugal nerves from the cerebro-spinal system carry vaso-dilator fibres. They act by inhibiting the activity of the ganglion-cells in the nervous plexuses around the vessels, thus causing a diminution of the vascular tonicity. They are to be considered, not as constant, but as occasional antagonists of the vaso-constrictor nerves. Goltz held that the dilatation which occurs after section of nerves is due, not to paralysis of the vaso-constrictors, but to stimulations of vaso-dilator fibres; and in his original experiment on the chorda tympani, Claude Bernard showed that both constriction and dilatation of vessels may be obtained not only directly, but by reflex action.

Dr. Handfield Jones has shown that irritation may be reflected from the fifth nerve on to the vaso-motor nerves of the arteries supplying the skin of the face, and that these, in consequence of the morbid impression, become dilated, not contracted as they nominally should according to the laws of reflex action.

The lesions to which the sympathetic is subject are manifold. In a large number of these coarser lesions, the influence is not that of the sympathetic on disease, but of disease on the sympathetic. Both in ataxy and in tetanus, redness of the semilunar ganglia has been observed. Inflammation of the semilunar ganglia has been associated with headache, hypochondriasis, vomiting, and death from marasmus; inflammation of the left portion of the solar plexus with pertussis, spasmodic vomiting, and convulsions; inflammation of the ninth and tenth thoracic ganglia, after retrocession of an exanthematous disease, with opisthctonos; vascularity of the sympathetic nerves in the chest, and of the semilunar ganglia, with tetanus; great increase in the size of the lower cervical ganglia with cretinism; great increase in size of all the ganglia with idiocy; enlargement of all the abdominal sympathetics and of the splanchnic nerves with diabetes; increase in size of the semilunar'? ganglia with a case of tuberculous suprarenal capsule; increase of size of the same ganglia with cancer of the stomach. One of the semilunar ganglia was of the size of a filbert, and cartilaginuus, in a case of madness. The abdominal ganglia have been found large, lobulated, yellowish, and of firm consistence in chronic peritonitis. Cholera has been sometimes associated with inflammation of the solar plexus and of the semilunar ganglia.

According to Pio Foá, lesions are most commonly seen in the cervi$\mathrm{cal}$ and the abdominal ganglia. These lesions are-simple and fibrous atrophy, hyperæmia, sclerosis, fatty and pigmentary infiltration, amyloid degeneration, accumulation of colourless blood-corpuscles, and the presence of micrococci in the blood-vessels of the ganglia. These changes are well marked in syphilis, leukæmia, a high degree of cachexia, pellagra, tuberculosis, cardiac disorders, and infectious diseases. 
Fournier thinks that the sympathetic system is affected in secondary syphilis, as shown by variations of temperature, by sweating, and even by epileptic seizures; but his views seem founded more upon symptoms than on pathological anatomy.

The most usual lesions are pigmentation, colloid degeneration, with proliferation of endothelial cells, and secondary fatty metamorphosis ; interstitial hyperplasia, leading to atrophy and sclerosis of nerve-elements; and such lesions are more than enough to modify vaso-motor functions, and, according to their seat, to lead to very various morbid phenomena.

Morselli has found fatty degeneration and atrophy"of ganglion cells, with thrombotic obliteration of the vessels of the cervical ganglia; whilst, in a case of unilateral swelling, Ebstein has seen very dilated and varicose blood-vessels in the ganglia of the affected side. Colomiatti has seen lipoma of the sympathetic, and a tuberculous nodule in the last left dorsal ganglion but one of the great sympathetic, and a similar nodule on the communicating bands between this ganglion and the one above it. The same observer has seen cancer of the semilunar ganglia, compressing and atrophying the cells, entering into the nervetrunks and the substance of the neurilemma.

In sunstroke, hæmorrhage has been found in the upper cervical ganglion. Hilton saw attenuation of the right side of the heart, associated with shrivelled ganglia of the same side of this organ; whilst Giovanni considers fatty degeneration rare, but thinks that the sympathetic resents most of the diseases affecting the whole system, and that lymphatic infiltration of its ganglia is the expression of the peculiar influence which it suffers. This condition, he believes, he has found in pleuropneumonia, cardiac disease, tuberculosis, aneurysm of the aorta, diffused tumour, acute and chronic nephritis, chronic enteritis, cirrhosis of the liver, suppurating ovarian cyst, typhoid fever, cancer, puerperal peritonitis, puerperal fever, pyæmia, pellagra, syphilis, scurvy, leukæmia splenica, tabes mesenterica, tabes dorsalis, erysipelas, epidemic cerebro-spinal meningitis, hydrophobia, exophthalmos, angina pectoris, and diphtheria.

An atrophic shrinking of the nerve-cells proper, and a calcareous mass occupying the position of the inferior cervical ganglion, were found by Dr. Shingleton Smith in a case of exophthalmos. A late observer has found very definite lesions of the sympathetic in phthisis; dilatation of vessels in the ganglia, proliferation of the connective tissue, hyperplasia of the epithelial covering of the capsules of nervecells, atrophy and pigmentation of cells. In chronic cases, there was found an enormous development of dilated blood-vessels; the external membrane of the ganglia (with its internal processes), the external capsule of the ganglionic nerve-cells, the neurilemma of the nervefibres, and the tunica adventitia of the blood-vessels, were all greatly thickened. This was especially the case in the inferior cervical ganglion. The lesions of the sympathetic on the healthy side, in unilateral phthisis, were but slight. The sympathetic lesions may be considered secondary. But the ganglionic affection, though caused by the previous disease, may itself induce some of the phenomena of the disease, as the hectic flush of the cheek and the facial perspiration, even if it do not determine certain trophic changes in the lungs.

The investigations of Dr. Saundby, one of our most accurate pathologists, seem to prove that the relationship between the renal lesion in Bright's disease, and the changes observed in the ganglia and surrounding connective tissue, must be regarded as secondary rather than primary.

It is easy to understand that such lesions, secondary in themselves to the diseases with which they are connected, yet impress upon the course of the disease certain special phenomena. Flushing, sweating, tinnitus, headache, faintness, palpitation, constipation, diarrhœa, vascular congestion of the intestines, diuresis, etc., are all examples of this.

These facts are more than mere matters of pathological interest. Given a recognisable lesion of a sympathetic ganglion or nerve, we find certain phenomena following this as a sure consequence. It is nature's own experiment to teach the physiology of the sympathetics. But, on the other hand, given these same phenomena, without a coarse lesion of the sympathetic nerve or ganglion, are not we justified in saying that they depend on a morbid condition of these structures, even though such a condition cannot be recognised by our means of investigation? This is what happens frequently. The common distinctions between organic and functional disease of the sympathetic is only an unscientific method of expressing this thought. A ganglion, or a series of ganglia, apparently healthy, is changed in some occult way by the sun's rays, by the circulation of blood altered from its normal condition, by what we call "irritation" carried to it from disease in a distant organ, or by emotions. We cannot doubt that these influences change in some way the equipoise of the ganglion : for, as their result, are seen phenomena precisely corresponding to the effects of coarse experiments upon the sympathetic in animals, and of easily recognised lesions upon these organs in man. We see the starting-point of the irritation, the channels by which the irritation is conveyed, the consequence of the irritative action beyond the ganglion; but the absolute condition of the ganglion itself, in so far as it differs from its state in health, is incapable of being in all cases demonstrated. The irritation may arise from some portion of the same nervous system, or from any portion of the cerebrospinal. It may be reflected only on its own fibres, or upon cerebral or spinal nerves. The exodic response from the ganglia may be carried back solely to the point of origin, or in very various directions to many organs, and through many and various channels. The reflected effects may be motor, sensory, or vaso-motor, or all together ; and yet the ganglionic centre of this reflex arc may seem, even microscopically, to be unchanged.

That change of some kind ensues cannot be doubted. All that can be said is, that it is a change so minute as so far to baffle our means of research, or so transient as to pass away before the possibility of post mortem examination.

In a very interesting paper read at the International Medical Congress last year, Dr. Woakes speaks of the inferior cervical ganglion as a correlating nerve-centre. He has formulated anatomically data that are occurring to all practitioners every day of their lives; and his paper seems to me to afford additional evidence, both of the independence of at least the vaso-motor portion of the sympathetic, and also of its multifarious connections with the cerebro-spinal system. He shows the association between injury of the fibres of the brachial plexus and loss of consciousness. The shock resulting from the concussion of the brachial nerves is propagated to the inferior cervical ganglion, and thence reflected as a wave of vessel-dilatation to the vertebral artery. This dilator wave is appreciated first of all in its peripheral branches, producing an immediate large accession of blood to them. This effect on the internal auditory branch of the dilated vertebral artery, the sudden tension of the intralabyrinthian fluid, produces the phenomena of falling and unconsciousness. Through the inferior cardiac nerve of this ganglion, great agitation of the heart may be induced. The vertigo attending indigestion under certain circumstances is an illustration of the same correlation, sometimes with, sometimes without, a sensory aura. This vertigo may also be associated with mottling of the hands and forearms, due to congestion of the superficial arterioles.

This note on the correlation of the ganglia can be expanded almost ad infinitum. The syncope induced by a blow over the solar plexus, the palpitation and even faintness consequent on indigestion, the pain, vomiting, and depression of spirits set up by the passing a gall-stone, the transient hemicrania produced in some people by the presence of ice in the stomach, the flux from the intestinal vessels as a sequence of the irritation of some foreign body in the canal, the numerous phenomena-motor, sensory, and vascular - associated with renal calculus, the peculiar pulse of peritonitis, the collapse of perforating ulcer of the stomach or intestine, perhaps the increased circulation of blood in the liver and the increased secretion of bile following injury to the solar plexus - are all instances of a similar fact with reference to this great nervous centre and its immediate branches.

But it remains for the hypogastric plexus to afford the most suggestive illustrations of this correlation. Without saying that all hysteria owns an uterine origin, the phenomena that are sequential to the multifarious lesions of the uterus and its appendages can be seen to im. plicate most of the important organs of the body. The deep-seated sense of pelvic uneasiness, nearly similar in position and sometimes equalling in intensity the sacro-coccygeal pain attending piles, the paresis of intestine evidenced by meteorismus, the increased flow of limpid urine, the vomiting, the hiccough, the frequent diarrhœa, the palpitation, the faintness, the sighing respiration, the globus, the difficulty in deglutition, the blushing, the dilated pupil, the tears, the tinnitus, the excitation of the emotional area, the occasional epilepsy, melancholia, and mania, to which such patients are liable, are all examples of afferent irritation carried to the solar plexus, and thence, from ganglion to ganglion of the sympathetic chain, to the three cervical ganglia-thence to the eye, the cerebral vessels, and the medulla oblongata.

The same causes, reflected from sympathetic ganglia to the sensory centres, induce the various neuralgix met with under these circumstances; whilst a similar irritation of a ganglion sending efferent nerves to a motor centre will lead to hysterical tortic sllis; to those manifold tonic contractions of limbs or of groups of muscles frequently seen, especially in hysterico-epilepsy; or to various clonic phenomena, such as the rapid twinkling of the eyelids.

Nor is the reverse side of the picture without its peculiar in- 
terest. Not only may uterine irritation induce emotion, but emotion may in itself induce all kinds of vaso-motor irritation or paresis, especially the latter. The emotion of terror, affecting primarily vaso-motor centres in the cortex of the brain, sends a paretic effect down the various ganglia in the body, besides inducing its own particular conditions on the cerebro-spinal motor nerves. It affects especially the sympathetic cardiac nerves, the accelerating nerves of the heart, leaving the chief influence on this organ to the inhibitory branches of the vagus, which this nerve derives from the spinal accessory. Blanching of the face, and even syncope, may result. By its paretic effect on the splanchnic nerves, the great inhibitory nerves of the intestine, the peristaltic movement of the gut is morbidly increased, and the tendency to evacuation of the bowels results, so frequent with young recruits on first going into action.

Blushing, a sense of heat, and sometimes arterial pulsation, are ordinary efiects on the face in certain emotional states-anger, joy, shame, bashfulness, and intimidation. Vulpian believes the emotional centre to reside in the annular protuberance. From this centre, the medulla oblongata and spinal cord are influenced; and thus, according to circumstances, may be caused either contraction of certain muscles of animal life, or a sudden state of muscular feebleness, or disturbance of the cardiac movements, or secretory alterations in the intestines, or, coincidently with these, some modifications of vascular tone in certain regions of the body, especially of the face and head.

Blushing is not always limited to the face. In nervous women, it is seen on the anterior superior part of the thorax as far as the mamnix, and even to the lower part of the sternal region, and above the clavicles, and over the shoulders. In a patient now under observation, the corjunctivæ redden, tears flow, a dark purplish blush suffuses the face, the neck, especially below the ears, and the whole of the anterior chest to the nipples, if she merely think of anxieties that she may, but very improbably will, have to undergo. In this case, the purplish blush is not in all places synchronous, but various islets of colour are separated by regions of skin of ordinary whiteness, and gradually coa. lesce ; illustrating the observation of other physicians, that the skin is mapped out into small vascular territories, more or less independent of each other, each under the influence of distinct vaso-motor nerves, or even of peripheral vaso-motor ganglionic centres. In a nervous man, whose case is recorded by Vulpian, blushing occurred on expesure, not only over the above-named regions, but over the anterior surface of the abdomen and the upper half of the thighs.

Paralysis of the splanchnic induces hyperæmia of the capillaries of the contorted renal tubules, with albuminuria and diuresis.

Emotional influence may reach the seven lower dorsal ganglia, from which the splanchnic nerves are derived, or with which at least they are connected, from the vaso-motor centre in the medulla oblongata by a branch that descends through the osteo-fibrous sheath which incases the vertebral artery, and thus by way of the inferior cervical ganglion; or the channel of conduction may be by way of the cord also.

One sees, therefore, anatomically, by how small an expenditure of force emotion may partially paralyse the cardiac and splanchnic branches of the sympathetic.

The vesical plexus, containing a few motor but more sensory and refiex acting fibres, is constantly affected reflexly by irritation from adjoining organs. But its chief motor nerves are derived from the supe rior and inferior genito-spinal centres in the spinal cord; and the effect of emotion on the bladder, causing frequent micturition, one of the most usual results of terror, and probably experienced by a large num. ber of candidates for examination, is due to paralysis of the purely sympathetic plexuses, allowing undue play to the motor influence of the sacral nerves from the centrum genito-spinale.

This reversal of the course up and down the sympathetic, the emotion acting directly downwards on the various ganglia, the uterine irritation acting upwards, in what may be called a series of reflex arcs, form the main difficulty in the diagnosis of the cause of that aggregate of morbid phenomena that, as a convenient expression for formulating our ignorance, we term hysteria, and has been well formulated by Mr. de Berdt Iovell. And putting aside those cases of crying and laughing, of globus, of hysterical vomiting, clanging cough, et hoc genues omne, which are really under the influence of the will of the patient, or are indulged in from a selfish desire to concentraie all attention upon herself, the Protean disease under discussion is, in the more numerous cases that are likely to fall under the notice of a physician, a very real ailment. Cases that are frequently the opprobria medicina are so, because the exciting cause is not found, sometimes scarcely sought for ; and Dr. Tilt, and those who think with him, have done good service in reminding us that the name given to the disease by our predecessors was founded very largely on ascertained fact. Scherschensky finds that the uterine plexus contains the most important, if not the only, motor nerves, which can produce actual movement of the uterus on stimulation of their peripheral ends. Stimulation of the central ends produces only violent vomiting.

Nor is it only in strictly physical phenomena that this pelvic irritation manifests itself. The somnolence, the trance, the contracture, the clonic convulsion of the hystero-epileptic, are evidently due to reflex paresis, or reflex irritation. Do not the more purely mental phenomena own the same origin? Is not emotion a brain-discharge, at least as much as epilepsy? and the suspicion, the anger, the fear, the melancholy, seen in various phases of this strange condition, are surely due to the variation in the calibre of the arteries, and consequent change in blood-supply, due to the influence of the cervical ganglia on the circulation of the brain.

Many people shrink from the word "materialism." It is because the word has been used to represent so limited an outlook. To recognise that the brain acts in accordance with the great laws of nature; that this thought and that emotion are, so to speak, discharged in relation to the amount and quality of the circulating fluid and the regions it traverses, is not materialism in a bad sense, but simply a statement of the method by which these laws work in correlation. And when we see vaso-motor effects manifested in the motor area of the brain, and coincidently with these the psychical phenomena just mentioned, it would be unscientific not to recognise a similar condition, as the exciting cause of these emotions, obtaining in a non-motor cerebral area.

Differing in degree, rather than in kind, Hypochondriasis stands out for both sexes, and especially for our own, as a term that includes selfconcentration, irritability, suspicion, melancholy, and various physical phenomena. All honour to the old Greek physicians who associated as cause and effect morbid action of the liver with depression of spirits. The blood may thus, in an impure state, affect every vasomotor centre in the brain or elsewhere ; or some chronic form of indigestion will give rise to a morbid impulse, often reflected to the inferior cervical ganglion, and so through the cardiac nerves to the heart, or to the upper and middle cervical ganglia, and from them to the cerebral circulation, but most frequently to the thoracic ganglia, and from them along the splanchnic nerves to some other portion of the solar plexus, leading to distension of the colon, to borborygmus, to constipation, or diarrhœa. In each case, the influence is reflex; but the reflex arcs may be somewhat complicated, or at least multiple. The starting-point is by no means always the liver. Cancer in any portion of the frame, especially of the peritoneum or of the abdominal viscera, seems frequently to be the exciting cause. The various phenomena associated with the sympathetic chain will readily be recognised by all practitioners as the symptoms most usually complained of by sufferers from hypochondriasis. Tinnitus, vertigo, confusion in the head, faintness, palpitation, a sense of impending dissolution, dyspepsia, constipation, meteorismus, with various mental delusions and extreme depression, irritability, or torpor of mind, are common enough.

Abnormalities of taste and smell, hallucinations of hearing, the falsity of which may or may not be recognised, anæsthesiæ, hyperæsthesiæ, paræsthesiæ, to say nothing of the numerous sensations con. nected with the spermatic and hypogastric plexus, must all be explained by reflex irritations of a similar kind. Perhaps the main difference between these reflex arcs, and those in which the centre is wholly cerebro-spinal, lies in the fact that one afferent irritation in the domain of the sympathetic is enough to set in action many efferent results. It may be so slight, that it is carried to the first ganglion in its neighbourhood, and be reflected back either to the seat of irritation, or to some spot in its immediate vicinity. It is almost certain, indeed, that this effect is invariably produced on the vaso-motor nerves of the part first affected. But in many cases of hysteria and hypochondriasis the irritation does much more; it may affect all the ganglia above mentioned in the way described; it may miss the upper and concentrate its action on the middle, or more specially on the inferior cervical ; or the efferent effect may act only on the splanchnic nerves, or without touching them, on the semilunar ganglia, or on some one or more of the plexuses connected with them. But in these morbid states, as in some others, the most common reflex arc seems to be made of irritation starting from the solar plexus, running up to the inferior and middle cervical ganglia, or even higher, causing a reflex paralysis of the middle and inferior cardiac nerves, and permitting therefore the inhibitory action of the vagus to act uncontrolled by these nerves and to diminish the action of the heart.

The reflex action of such conditions is illustrated by Dr. Wilks's cases of sympathetic mania from the presence of a tumour in the abdomen, from adherent omentum, from unilateral hernia, from misplacement of the colon, and from cæcal abscess.

Of other forms of neurasthenia, the description would be almost 
the same. In several cases, in my own practice, there has been a peculiar subjective sensation all down the spinal cord, never amounting to pain or paralysis, but causing a restlessness combined with a lack of energy that is very distressing to witness. Calabar bean has at times served to promote recovery.

The nervous terror of such patients is a marked feature; terror of horses, of crossings, of fire, of water, sometimes associated with that shrinking from meeting other people that is seen in other nervous com. plaints. The causation of these symptoms is, in a word, starvation of the nervous centres, either by calling too much on them in the way of work, of anxiety, of sexual indulgence, of fatigue of any kind, or by absolute starving these centres of the fatty material requisite. The mechanism is by way of the vaso-motors, as is shown by the remedial agents by which such patients are benefited, as well as by the nature of the phenomena themselves.

This reflex mode of action can scarcely be left out in the consideration of any one point in which the sympathetic is involved. By no means is it least in the great questions of inflammation.

From the masterly Lectures on Inflammation delivered here last spring by Dr. Burdon Sanderson, one may gather certain dicta, which amount almost to axioms.

I. The statement of Lister, that stasis is not due to alterations of the circulating blood, but to a change in the channels, through which it has to pass.

2. The temperature of an inflamed part never exceeds that of the rectum.

3. Cohnheim has shown that the increased temperature of external organs, when inflamed, depends on the activity of the'circulation.

4. Determination of blood is a frequent precursor of inflammation, but it is not a part of it.

5. Reflex congestion produced by stimulation of sensory nerves is not the same as inflammation.

It has been known, since Claude Bernard's experiments, that division of nerves belonging to the system of organic life gives rise to three great classes of phenomena, acceleration of the passage of blood through the vessels, increase of temperature, exaggerated activity of the secretions.

The physiological history of inflammation is briefly this :

I. Some source of irritation, cold, a blow, a burn, a septic focus.

2. The centripetal nerves, whether sensory or not, which are within reach of this irritation, are excited more or less violently.

3. These nerves transmit to the vaso-motor centres of the region the excitation which they have undergone.

4. The tonic activity of these centres is disturbed, and suspended more or less completely.

5. Hence follows cessation or diminution of the tone of the vessels that are subordinate to these centres.

6 . Consequently, more or less considerable dilatation of these vessels occurs.

7. But this vaso-motor disturbance can only be considered as favouring the development of inflammation. It is only secondary in importance, and does not suffice of itself to make up the 'phenomenon that we call inflammation. It places the vessels in a condition for easily and necessarily receiving more blood; it induces stasis of circulation, and offers facilities for the emigration of leucocytes; but the initial phenomena of inflammation consist in the disturbance of the intimate nutrition produced in the organised living tissue. The vitality of the cell having been gradually lowered by the previous state of its nutrition, it is thereby placed in a condition of vulnerability, and is ready at any moment to respond to morbid impressions. These may be reflex, as the impress of cold, causing pneumonia, it may be ; or direct, as from the presence of morbid germs in the blood. And the vaso-motor action of the vessels, which, without this previous alteration of the cell-nutrition, would stop short at non-inflammatory congestion, is of enormous importance in determining the various stages and symptoms of the progress of inflammation, although independent, and unconnected directly with the initial phenomenon.

Some of the capillaries are blocked early by thrombi, others that are permeable are dilated, and the course of the blood, instead of being continuous, as in the normal state, becomes jerky, as in the arteries and thence is carried, partly at least, the sensation of pulsation experienced in an inflamed region under certain circumstances.

As to the mechanism by which the vaso-motor centres of the bulbospinal axis, or at least of the vaso-motor ganglia, induce, under the influence of the irritation transmitted to them, a dilatation of vessels in the inflamed region, it probably consists in a suspension of the tonic activity of these centres. At any rate, a reflex irritation of vasodilator nerves is in most cases difficult of proof.
This theory of inflammation is practically a mere statement, in other words, of the dictum of Vulpian, that, in pneumonia, besides the mechanism of the cold impressions influencing in a reflex way the nutrition of the lungs through the nervous centres, there is need to admit, first, a general predisposition to inflammation ; and, secondly, a local predisposition, which renders the respiratory organs more sensitive to the reflex action of cold than other parts of the body.

But although vaso-motor paresis has little or nothing to do directly with the initial phenomena of inflammation, there are numerous instances of indirect action. Why do we look with grave suspicion on pulmonary congestion, or a similar condition in any other portion of the system? Is it not that a region so affected is peculiarly liable to take on active mischief? that is, a part that for a time has been affected only by means of partial paralysis of the vaso-motors, can very readily be found to be the seat of inflammation. It was this prominent fact that probably misled observers as to the role of the vaso-motors in inflammation. Congestion so often passes into inflam. mation, that the former was supposed to be the cause of the latter. But the explanation of the connection is that given above. A congested part becomes gradually of necessity a part in which nutrition is badly performed. The affected spot, becoming less and less perfectly nourished, is ipso facto more or less vulnerable to influences external to itself ; in other words, it is predisposed to inflammation. The external influence arrives; modifies directly or reflexly still further the nutrition of a part already vulnerable, already possessing unstable cells ; and the predisposition, the external influence, the modification of nutrition by the exciting cause, and the vaso-motor paresis, make up the necessary factors in the causation of inflammation.

This slow predisposing influence of congestion is markedly increased if it result in œdema. The vaso-motor nerves are implicated in causing œdema in more ways than one. Paralysis of vaso-motors dilates the arteries, and fills them with blood. The capillaries become abnormally distended, passively dilated, and, in the case of any hindrance to the nervous circulation, œdema results. Or œdema may be reflex, following a dilatation of vessels due to reflex sensory irritation. The capillaries may remain patent, or be thrombotic; and we have traumatic irritation of centripetal nervous fibres, suspension of tonic activity of certain vaso-motor centres, paralytic dilatation of the muscular tissue of the vessels, diminished vis-a-tergo in the veins, and so adema. Or, again, reflex paralysis of vessels, with enfeebled vis-atergo, gives rise to a relative intravenous stasis, an increased pressure on the capillaries, and issue of liquid from the vessels. From whatever cause, the water-logging of a tissue by œdema must necessarily interfere with its nutrition.

This part of our subject can scarcely be left without a few more words on congestion. M. Notta observed conjunctival redness in thirty-four of sixty-one cases of neuralgia of the fifth nerve. This congestion may extend over half the face, and even inside the mouth. It may be explained by the hypothesis of vaso-dilators; but, as this cannot be universally proved, it is enough to say that the transmission of the excitation along the sensory centripetal nerves to the vasomotor centres of the regions where these nerves are distributed, may suspend the tonic activity of these centres, so as to cause a paralysis of the vessels of the corresponding regions. A similar reflex congestion may be occasionally seen in connection with neuralgia of other nerves.

The congestion of the second stage of ague owns a somewhat different causation. It is due to a dilatation of the vessels of the integument, that depends on a certain degree of vaso-motor paralysis, succeeding the stage of excitation of the cutaneous nerves in the first stage. This is not owing merely to fatigue of the nerves, as the dilatation is in no proportion to the duration or intensity of the cold stage, but to a special modification of the vaso-motor apparatus, produced directly or indirectly by the morbific agent. Fatigue of nerves may in many cases add to this. Vulpian, whose views on congestion I have followed pretty closely, attempts to explain the specific congestion of the exanthemata in a similar way, as a dilatation of the subepidermic vessels; and he says that, in measles, the eruption seems to show that the vaso-dilator cause acts on sets of neuro-vascular territories, distinct one from another; and that congestion of the internal viscera, the lungs for instance, in typhoid fever, is due to a functional modification of the vaso-motors of the lungs, similar to that of the vasomotors of the skin ; whilst, although the fulness of the sple en seems to depend on a proliferation of the cellular elements in that or gan, yet its rapid variation in size in ague and in typhoid fever must be induced by successive weakness and activity of the vaso-motor apparatus of this organ.

Nor can I forbear mentioning the congestion of the cheeks in pneumonia 
from reflex dilatation of the vessels of the cheek, unilateral pneumonis being often associated with unilateral congestion of the cheek; or the occasional phenomenon, in inflammatory disease of the lungs, of the arm of the affected side being hotter than the other, probably from reflex irritation carried to the bulbo-spinal axis and reflected along the brachial plexus, and the vaso-motors included in it. Of the same order is the congestion of internal organs, notably of the intestinal mucous membrane, following burns on the surface of the body. Ulceration of the duodenum is not unfrequently met with as a sequence of such burns and the mechanism is centripetal irritation from the skin to the spinal cord, reflected down to the semilunar ganglia and the subordinate vasomotor nerves to the intestine.

In diseases accompanied by pyrexia, there is no necessary connection between the state of the cutaneous vessels and the sweat-glands. In the third state of ague, the ressels seem in the same state as in the second, and jet sweating occurs. Probably, in the normal state, fibres from the sympathetic exercise a moderating action on the secreting work of the sweat-glands; when these fibres are paralysed, then hyperidrosis occurs.

Paralysis of the vaso-motor centre in the medulla oblongata by injury causes decreased production of heat, and probably always increased dis. sipation of heat, depending on the fact that the medullary centre dominates the vessels all over the body, and that general dilatation of all the vessels produces a sluggishness in the movements of the blood in all parts of the body. Increased heat of blood cannot, therefore, depend on this general paresis of all the vaso-motors, but must be due to affection of the controlling inhibitory heat centre or centres, that have been found by experiment to lie above the medulla oblongata.

What is known about the influence of the sympathetic on sweating has been mainly observer in cases of unilateral byperidrosis. Eulenburg and Guttman have remarked that, after sectinn of the cervical sympathetic-in one case in the left sympathetic-there were very varicose and dilated vessels, which perhaps, when full, pressed on some of the sympathetic nerve-elements, and so paralysed them; also that, by galvanisation of the cervical sympathetic, the secretion of perspiration in the arm is increased. They think that this is due to currents entering the brachial plexus or the spinal cord, and has nothing to do with be sympathetic proper.

Unilateral ephidrosis is sometimes seen in exophthalmic goître. Schwa. bach records a case in which pressure on the cervical sympathetic was associated with heat and redness of the right side of the face, and unilateral sweating on the least exertion. In Seguin's case, there was normal perspiration on the left side of the face, whilst the right side was absolutely dry, and here the right symathetic was adherent to the sheath of the vessels. In Ebstein's case of unilateral sweating, there were very dilated and varicose vessels in the ganglia of the affected side.

In Seeligmiller's case of a woman who had had right ephidrosis during the whole of life, and in whom all the symptoms of paralysis of the right cervical sympathetic were manifested, there was found after death sclerosis and fatty degeneration of the right cervical sympathetic.

We find that sweating follows paralysis of the sympathetic, whilst vaso-motor paralysis, sufficient to cause extreme dilatation of vessels, is not necessarily associated with sweating. It is certain, therefore, that one of the functions of the sympathetic is that of inhibiting overaction in the sweat-glands, of maintaining tone in these organs; and that hyperidrosis depends, not on the vaso-motor nerves, but on secreting fibres emanating from the cord through the rami communicantes to the sympathetic ganglia.

Seeligmiller asserts that the position of the sweat -centre is doubtful. It resides possibly in the spinal cord, possibly in the upper surface of the brain. That the irritation that excites hyperidrosis may be reflex, is seen in those cases of extreme perspiration of the palms of the hands induced by indigestion; and instances of perspiring feet, not only hyperidrosis but osmidrosis, are not wanting, in which the existing cause is to be sought in abdominal and pelvic irritation.

Vaso-motor paralysis is not necessarily accompanied by the secretion of sweat, but the reverse statement does not hold good. The secretion paralysis of the vaso-motor centres that preside over the vascular tone of the sweat-glands, hamatidrosis, bloody sweat, may result. And in spite of cases of simulation and of red fungus, it is certain that such bloody sweat is met with, depending on paralysis of the sympathetic motor inhibiting the action of the sweat-glands, flus an intense vasomotor paralysis of the vessels of these organs, and such a consequent congestion as may lead to rupture of vessel.

The influence of the sympathetic on the nutrition of a part, and on its circulation, render it, to say the least, one of the chief factors in inflammatory disease of every organ of the body. The vaso-motor supply of the cerebral and meningeal vessels brings the brain and its membranes into close relationship with the cervical ganglia. Take general paralysis of the insane. The lesions found post morlem are many and various, but in all cases there may be found cerebro-meningeal hyperæmia, that has often proceeded to emigration of leucocytes, distension of vessels, impediments (chiefly thrombstic) to the circulation, irritative overgrowths of tbe connective nuclei of the walls of the vessels, and probably also of the neuroglia. This is only a somewhat more modern expression of Calmeil's dictum, that the pathological lesion is chronic inflammation of the brain, especially of the superficial part of the convolutions, the grey substance, and the meninges. In addition to the dilive ambitiers, which is sometimes wanting, and which, occurring in a few other morbid states of the brain, cannot be considered pathognomonic of general paralysis, early inequality of the pupils, a sense of fatigue that yet does not cvercome the tendency to wakefulness, irritability of temper, slight paralytic phenomena of speech, of prehension, and of locomotion, with great facility in the formation of bed-sores, would be some of the more remarkable sym. ptoms of this condition. Not only does the inflammatory character of the disease connect it with the sympathetic, not only can the intermission of the morbid phenomena be explained in no other way, but the inequality of the pupils has been thought by some to point to the same fact. My friend Dr. Thompson, of the Bristol County Lunatic Asylum, justly says, however, that the asymmetry of the pupils is due to the want of symmetry in the rate of progress of the cerebral lesion.

Myosis is a symptom that so often occurs in disease, apt, as it is, not only to assist a diagnosis, but frequently to increase the gravity of the prognosis, that it may be advisable to devote a few words to it. Contraction of pupil is seen to follow compression of the cervical sympathetic by tumour to the extent of paralysing the sympathetic branches. In a recent case of some obscurity, the diagnosis of aneurysm was ren. dered certain by the unilateral myosis. This symptom is seen in lesions of the pons Varolii, in sclerosis of the medulla oblongata, in disease of the cervical cord, especially in tabes cervicalis, and sometimes in progressive muscular atrophy. It is found in traumatic paralysis of the brachial plexus from simultaneous paralysis of the cervical sympathetic the vaso-motor fibres being unaffected.

With the exception of one or two ciliary nerves of separate origin all the branches destined for the iris and ciliary muscle proceed from the ciliary ganglion. What influence is exercised on the ganglion by each of the three nerves by which it is supplied ?

I. The oculo-motor has undoubted action on the sphincter of the pupil. The pupil becomes dilated and immovable in paralysis of this nerve. This nerve is the condition, sine yut non, both for reflex and accommodative movement of the pupil.

2. The filaments of the sympathetic acting on the pupil arise from the spinal cord, and pass into the anterior roots of the two lower cervical and six upper dorsal nerves. There is slight contraction of pupil on section of this nerve, and gradual dilatation on irritation of it in the neck. After division of the sympathetic in the neck, the upper part passes into fatty degeneration. The action of the sympathetic root consists in a persistent exaltation of tone of the radiating fibres. It is not proved that it acts on the accommodation. Irritation of the sympathetic in the neck causes contraction of the vessels of the iris. Dilatation of the pupil from irritation of the sympathetic nerves is not due to contraction of vessels (the diminution of blood in the iris lessening contraction of the sphincter muscle), but depends on contraction of the radiating fibres of the iris.

3. The influence of the fifth nerve is doubtful, but it is probably sensory. As a motor influence it may act on the ciliary ganglion, either to increase the action of the fibres of the oculo-motor, or to diminish that of the sympathetic.

If these views are correct, and they are those of Donders, the position of the sympathetic in the causation of myosis is not a very im. portant one. Certainly, in general paralysis of the insane, where the lesions are largely intracranial, the myosis is due to irritation of the oculo-motor nerve rather than to the paralysis of the sympathetic; and if, in addition to this state of the third nerve, there be irritation of the fifth also, the effect of the sympathetic would be rendered nil. The influence of the sympathetic on the pupil can only be seen when neither of the other nerves supplying the ciliary gauglion are irritated or paralysed. The intermissions of general paralysis depend on the greater or less congestion of the brain or its membranes. Vulpian, indeed, goes further, and suggests that many of the so-called apoplectic attacks in this disease are not due to overdistension of the ressels, but to anxmia of parts of the brain, such anæmia being the result of reflex vaso-constrictor phenomena. The foci of white softening sometimes 
found in general paralysis may have this origin, but far more frequently is it the sequence of thrombotic blocking of minute arteries.

A similar reflex constriction of vessels may occur in tuberculous meningitis, or in tubercle of the brain, from the irritation of the foreign growth; but this theory is unnecessary, as the interference with the calibre of the vessels is fully accounted for by the early growth of tubercle on their adventitia.

Nor can more than the ordinary vaso-motor influence be traced in most of the other morbid conditions to which the cerebro-spinal nervous system is liable. In meningitis, in mania and melancholia (except that these latter may show remissions) in hæmorrhage, in softening, in sclerosis of the brain and cord, in inflammation of the cells of the anterior horns or in atrophy of the same, the sympathetic has not much to do directly. In one disease just mentioned-progressive muscular atrophy - the fact that the lesion is due to a malnutrition of cells of the anterior cornua, induced by a condition of the nutritive vessels rendered morbid by an abnormal state of the vaso-motor nerves, is probable, but certainly has never been demonstrated. It is, however, only fair to say that Sir Charles Bell thought the sympathetic was concerned in this disease, and that Jaccoud shares his views; and that, besides the lesions of the anterior horns, the cervical ganglia of the sympathetic have been found converted into fat, whilst the raised temperature, in the early stages diminished later, the increased perspiration, the atrophy of the layers of skin, the painful swelling of joints, and sometimes the contraction of one pupil, all point to some sympathetic connection. In pseudo-hypertrophic paralysis, one case has been attributed to paralysis of the sympathetic; but from the study of the physiology of the sympathetic, the course of the disease, the post mortem appearances, and the sufficiency of other explanations for the lesions, the conclusion is almost necessary that the sympathetic has little or nothing to do with this disease. It is quite another question whether progressive muscular atrophy has not some influence on the sympathetic. Myosis in this disease is rare; but, when it exists, it may depend in some sort on the cutting off more or less of the influence of this nerve from the ciliary ganglia, so that, its inhibitory effect on the contraction of the pupil being removed, the oculo-motor acts with great intensity. But this and all other sympathetic phenomena in progressive muscular atrophy seem to be secondary in point of time to the disease itself.

In sunstroke, the primary condition is twofold : paralysis of the inhibitory heat-centre, and paresis of the chief vaso-motor centre on the medulla oblongata. The loss of tone in the capillaries and small arteries, and the consequent congestion, is especially seen in the lungs, and forms an important element as to the peril of the patient. Hæmorrhages have been found in the ganglion cervicale supremum.

In epilepsy, there is much to be said about vaso-motor influence, although the opposite view has been taken by distinguished Fellows of this College. Meynert believes that in epilepsy the hippocampus major is a vaso-motor centre, irritation of which causes spasm of vessels, and so epileptic convulsions. Nothnagel considers vascular cramp an essential factor in all epileptic seizures. Binswanger says that, in a typical fit, excitement of the convulsive centre and of the vaso-motor centre are co-ordinated. If the excitement of the vaso-motor centre exist alone, there is le petit mal. If excitement of the convulsive centre exist alone, we have those rare cases of motor epilepsy, convulsion without loss of consciousness. Most frequently, both centres are excited together.

The influence of emotion, especially the terror at the sight of another person in an epileptic fit, seems so prominent in this disease, that this alone points to a vaso-motor basis.

Dr. Gowers, however, states his case clearly enough. The three views most in vogue are these.

I. Epilepsy is simply a disease of the vaso-motor centre in the medulla oblongata, setting up vaso-motor spasm affecting particular arteries, and thus causing local cerebral anæmia, which induces the discharge from the hemispheres. This theory is held by few.

2. Convulsion depends on discharge of motor or convulsive centres in the medulla oblongata; while loss of consciousness is the result of arterial spasm in the hemispheres, due to the action of the vaso-motor centres in the medulla.

3. The view of Dr. Hughlings Jackson; that the local discharge in the brain excites at the spot arterial contraction, and this determines the spread of the discharge.

To this Dr. Gowers objects, that pallor of the face is often absent; that, when present, it is no proof of anæmia of the brain, but is probably due to a reflex contraction of peripheral vessels excited by the discharge in the brain; and that convulsion is not usual in cardiac syncope; that this third view is not needed, and is opposed to the fact, proved by experiment, that functional debility causes reflex dilatation, and not contraction, of vessels.

He would say that the phenomena of epilepsy depend on instability of resistance, rather than on any primary change in the energy-producing action of the cells. It seems open to question whether this somewhat negative theory suffices to explain all the various forms of epilepsy; whether, particularly, it demonstrates the mechanism of ie petit mal. On the other hand, Echeverria has recorded twenty-six cases of epilepsy, in almost all of which there was found sclerosis or fatty degeneration, or amyloid degeneration, or pigmental infiltration of the cervical sympathetic, and often two or more of these changes united; sometimes also a similar condition of the solar and other ab. dominal plexuses.

Again, although the extraordinary high remperature in the status epilepticus is not a proof of the implication of the sympathetic, and may be caused merely by paralysis of the inhibitory heat-centre in the brain, yet this great heat can hardly arise without some vaso-motor change, even if this very paralysis of heat-inhibition be not caused by anæmia of the centre from reflex contraction of its vessels.

The numerous instances of true epilepsy, caused by reflex irritation from distant organs travelling upward by way of the sympathetic, are not wholly explicable by the theory of instability of cells. The epileptic condition, consequent on irritation of the uterus and its appendages; the gastric epilepsy in men, of which Pomeroy speaks as answering to uterine epilepsy in women, are only some of the examples of this reflex condition.

The difficulties are the greater, inasmuch as all theories must be more or less hypothetical. It seems likely that the convulsive centre in the medulla is a minute corpus striatum for collecting, modifying, radiating convulsive motor phenomena from the cerebral motor area; that epilepsy with convulsion may depend on direct or reflex irritation of this centre, but far more frequently on some condition of cells in the cerebral motor area, that may well be termed instability; that these lesions and their consequent phenomena may be wholly independent of vasomotor disturbance; but that loss of consciousness, occurring either as an early symptom of the convulsive form of epilepsy, or as an independent pheromenon in le petit mal, owns as its cause anæmia of the non-motor area of the brain; an anæmia depending on vaso-motor irritation. Brown-Séquard's experiment, showing that compression of the cervical sympathetic was a valuable means against le petit mal, points to the truth of this latter proposition.

The whole question of optical delusion is more or less under the influence of vaso-motor action. In health, the impression of an external object is carried to the retina, and thence to the corpora quadrigemina. Thence it is transmitted to the angular gyrus as a sensory centre, and reflected on to the anterior lobes for perception. But in certain variations of the vascular tone of the vessels of the angular gyrus this centre seems to have the power of evolving optical delusions, wholly irrespective of external impressions. Many of the phenomena of febrile delirium, of delirium tremens, and of mania are produced by vascular congestion, or by anæmia, of the angular gyrus. Probably, too, the false sensations of optical impressions depend on a similar congestive condition of the gyrus, including many of the varieties of hallucination and illusion.

\section{[To be continued.]}

The Poison of Mushrooms.-Professor Ponfick, of Breslau, has lately made experiments on the common mushroom, of which the following are the results. All common mushrooms are poisonous, but cooking deprives them, more or less, of their poisonous qualities. The repeated washing with cold water which they usually undergo to clean them, takes away a portion of the poison, and boiling does the rest ; but the water in which they have been boiled is highly poisonous, and should always be carefully got rid of. Experiments made on dogs showed that if a dog ate one per cent. of its own weight of raw mush. rooms it fell sick, but recovered; if it ate one and a half per cent. the poison had a more violent but not fatal effect, and if it ate two per cent. it was inevitably fatal. The water in which mushrooms had been boiled was far more poisonous than even the raw mushrooms, while the mushrooms thus boiled could be taken witheut hurt to the amount of ten per cent. of the weight of the dog's body. Washing with cold water does not remove all the poison, so that mushrooms thus prepared are poisonous when taken in larger quantities. Dried mushrooms are still dangerous for from twelve to twenty days, and also the water in which they have been $b$ oiled. They require to be dried for at least a whole month, and are only rea!"y ' $\ni$ fe after four months' drying. 